\title{
Inhibition of MSP-RON signaling pathway in cancer cells by a novel soluble form of RON comprising the entire sema sequence
}

\author{
QI MA ${ }^{1,2}$, KUN ZHANG ${ }^{1}$, HANG-PING YAO ${ }^{3}$, YONG-QING ZHOU ${ }^{3}$, \\ SNEHAL PADHYE ${ }^{1}$ and MING-HAI WANG ${ }^{1,2,3}$ \\ ${ }^{1}$ Center for Cancer Biology and Therapeutics and ${ }^{2}$ Department of Biomedical Sciences, School of Pharmacy, \\ Texas Tech University Health Sciences, Amarillo, TX 79106, USA; ${ }^{3}$ Laboratory of Cancer Biology, State \\ Key Laboratory for Diagnosis and Treatment of Infectious Diseases First Affiliated Hospital, \\ Zhejiang University School of Medicine, Hangzhou 310003, P.R. China
}

Received January 18, 2010; Accepted February 24, 2010

DOI: 10.3892/ijo_00000642

\begin{abstract}
The RON receptor tyrosine kinase and its ligand macrophage stimulating protein (MSP) play a role in epithelial tumorigenesis. We report here a novel RON variant that antagonizes the RON-MSP pathway in various cancer cells. The variant is an $85 \mathrm{kDa}$ soluble protein from an mRNA transcript with an insertion of 49 nucleotides between exons 5 and 6 . The insertion created a stop codon leading to the formation of a RON variant consisting of the entire $35 \mathrm{kDa} \alpha$-chain and a $45 \mathrm{kDa}$ partial extracellular $\beta$-chain. The protein was featured by a sema domain, a hinge motif and a portion of the first IPT unit (designated as RON $\Delta 85$ ). RON $\Delta 85$ binds directly to MSP, forms MSP-RON $\Delta 85$ complex, and inhibits RON phosphorylation. RON $\Delta 85$ disrupts RON or RON $\Delta 160$ dimerization, prevents their phosphorylation, and attenuates downstream signaling events. The action of RON $\Delta 85$ is specific to RON and has no effect on MET and EGFR. In colon and pancreatic cancer cells, RON $\Delta 85$ inhibits spontaneous or MSP-induced Erk1/2 and AKT phosphorylation, which results in impaired cell proliferation and colony
\end{abstract}

Correspondence to: Dr M-H Wang, Department of Biomedical Sciences, School of Pharmacy, Texas Tech University Health Sciences Center, 1300 S. Coulter Street, Amarillo, TX 79106, USA E-mail: minghai.wang@ttuhsc.edu

Abbreviations: CHO, Chinese hamster ovary; EGFR, epidermal growth factor receptor; HGF, hepatocyte growth factor; IPT, immunoglobulins, plexin, and transcription factor; MAP, mitogenactivated protein; MDCK, Madin-Darby canine kidney; MSP, macrophage-stimulating protein; PSI, plexin-semaphorin-integrin; RON, recepteur, d'origine nantais; RT, reverse transcription; RTK, receptor tyrosine kinase; sema, semaphorin; si, small interfering

Key words: receptor tyrosine kinase, truncated variant, antagonistic activity, signal transduction, epithelial cancer formation. RON $\triangle 85$ also inhibits spontaneous and MSPinduced cell migration. We conclude that RON $\Delta 85$ is an antagonist to the MSP-RON pathway, which has potential for regulating RON/RON160-mediated tumorigenic activities.

\section{Introduction}

Macrophage-stimulating protein (MSP) activates the RON receptor tyrosine kinase, which regulates activities of epithelial cells including migration, proliferation, and survival (1). The MSP-RON pathway plays also a role in epithelial carcinogenesis (2). RON is overexpressed in many primary cancer samples including breast, colon, and pancreatic tumors (3). Overexpression has been associated with advanced clinical stages and poor prognosis (4). In mouse model, mammaryspecific RON expression causes the formation of highly metastatic tumors (5). Transgenic expression of RON in lung epithelial cells also results in lung tumors with features of bronchiole-alveolar carcinomas (6). These pathogenic phenotypes are manifested by RON-directed signals that regulate cell dissociation, motility, and matrix invasion (7). Thus, targeting the MSP-RON pathway has therapeutic potential. Currently, various approaches including therapeutic antibodies (8), siRNA (9), and small molecule inhibitors (10), are under intensive investigation. Results from these studies are promising with prospects for future clinical evaluation (11).

MSP-induced RON activation depends on the coordinated interaction between binding domains on the MSP molecule and ligand-recognition pockets in the RON extracellular sequences. Two binding moieties on MSP have been observed. The high affinity binding moiety is located in the serineprotease-like domain in the MSP $\beta$-chain (12). The $\alpha$-chain contains a weak binding moiety, which resides in the kringle domains of the $\alpha$-chain (13). Both sites are functionally required to activate RON for signaling events (12). Ligandrecognition pocket(s) on the RON extracellular sequences are currently unknown. RON extracellular sequences contain a sema domain followed by a cysteine-rich hinge (PSI), and four IPT (immunoglobulin-plexins-transcription) units (14). The 
sequences coding the sema domain are a stretch of 500 amino acids that are located on both RON $\alpha$ - and $\beta$-chain (14). Analysis of MET, a homologue of RON, have revealed that the sema domain contains a low affinity site for HGF binding (15). The high affinity HGF binding pocket resides in the fourth IPT of the MET B-chain (16). Using recombinant RON fragments containing the sema domain, it has been showed that the protein prevents MSP binding to RON and inhibits MSP-induced RON activation (17), suggesting that the sema domain may bind to MSP. Thus, the sema domain of RON represents not only an important structure for ligand recognition but also a critical requirement for receptor activation and signal transduction.

The study in this report is on the biological significance of a naturally occurring soluble RON variant containing the complete sema sequence. Our purpose is to determine if the sema domain of RON plays a role in interaction with MSP and acts as a potential inhibitor of RON-mediated tumorigenic activities. Since altered RON expression exists in many cancer cells, which is characterized by the presence of various shortened, spliced, or truncated mRNA transcripts (18), we used PCR techniques to isolate RON mRNA transcripts that are not translated but possess potential regulatory activities upon recombinant expression. One mRNA transcript was isolated from breast cancer Du4475 cell line. The synthesized protein is an $85 \mathrm{kDa}$ RON variant composed of a full $40 \mathrm{kDa}$ $\alpha$-chain and a partial extracellular $45 \mathrm{kDa} ß$-chain (designated as RON $\Delta 85$ ). Both chains are linked by a disulfide bond(s). Biochemical and biological analyses demonstrated that this novel RON variant directly binds to MSP and inhibits MSPinduced RON activation. It also regulated signaling events and tumorigenic activities mediated by RON and its oncogenic variant RON $\Delta 160$ in a ligand-dependent or independent manner. Our results highlight the potential inhibitory use of this novel RON variant to regulate tumorigenic phenotypes mediated by the MSP-RON pathway.

\section{Materials and methods}

Cell lines, antibodies and reagents. Human breast (Du4475, HCC1937, T-47D, MDA-MB-231, MCF-7), colon (HCT116, SW620, SW683 and HT29), and pancreatic (BxPc3 and Panc-1) cancer cell lines and CHO-k1 cell line were from ATCC (Manassas, VA). MDCK cells expressing RON (M-RON) or RON $\Delta 160$ (M-RON $\Delta 160)$ were used as previous described (19). Human recombinant HGF, pro-MSP, mature MSP, MSP $\alpha^{-}$, and $\beta$-chain were from Dr E.J. Leonard (National Cancer Institute, Bethesda, MD). Human EGF and anti-v5 antibodies were from Invitrogen (Carlsbad, CA). Cross-linker Sulfo-EGS was from Pierce (Rockford, IL). Mouse mAb $\mathrm{Zt} / \mathrm{g} 4$ against the sema domain of RON and rabbit IgG antibody against RON C-terminus were used as previously described (20). Antibodies to various signaling proteins including, ERGR, MET, Erk1/2, and AKT were from Cell Signaling, Inc. (Beverly, MA).

RT-PCR and RON $\triangle 85$ cDNA construction. Total RNAs were isolated from Du4475 or other cell lines using TRIzol (Invitrogen) as described previously (21). RT-PCR was carried out using $2 \mu \mathrm{g}$ of total RNAs with SuperScript kit
(Invitrogen). RON oligo-primers that cover the RON extracellular sequences were designed as previously described (21). The cDNA fragments were sequenced at the Texas Tech University DNA Sequence Core facility. The mammalian expression vector pcDNA3.1V5/His was used for RON $\Delta 85$ expression.

Establishment of transfected cell lines and purification of $R O N \Delta 85$. Transfection of CHO-k1 cells with plasmids was performed as previously described (20). Positive clones $(\mathrm{CH}-$ RON $\Delta 85)$ were selected and expended into cell lines. To purify RON $\Delta 85$, supernatant from $\mathrm{CH}-\mathrm{RON} \Delta 85$ cells was concentrated and then loaded on the Ni-NTA column. The eluded proteins were analyzed on $10 \%$ of SDS-PAGE, filtersterilized and used for further experiments.

Protein cross-linking. Cells at $1.5 \times 10^{6}$ cells in a $60 \mathrm{~mm}$ culture dish were treated at $37^{\circ} \mathrm{C}$ for 15 min with or without MSP $(5 \mathrm{nM}), \mathrm{RON} \Delta 85(35 \mathrm{nM})$ for their combination. Cross-linker Sulfo-EGS was added to the culture medium at a final concentration of $2 \mathrm{mM}$ for $30 \mathrm{~min}$ at room temperature. Reactions were stopped by washing cells with cold PBS. Proteins from cell lysates were analyzed by Western blotting using rabbit antibodies to RON followed by ECL visualization.

Immunoprecipitation and Western blot analysis. These methods were performed as previously described (22). Individual samples (350 $\mu \mathrm{g}$ protein/sample) were immunoprecipitated by respective antibodies ( $2 \mu \mathrm{g} /$ sample) coupled to protein $\mathrm{G}$ Sepharose beads. Western blot analysis was performed by using specific antibodies followed by ECL reaction. Results were recorded and analyzed using VersaDoc Imaging System (Bio-Rad).

Assays for cell proliferation and migration. These assays were performed as previously described (19). For proliferation, cells were cultured $\left(4 \times 10^{3}\right.$ cells/well) in triplicate in a $96-$ well plate and then treated with or without MSP $(5 \mathrm{nM}), \mathrm{RON} \Delta 85$ ( $35 \mathrm{nM})$ or their combination for 3 days. Cells were stained with Hema-3 stain kit (Fisher Scientific) and then lysed in $1 \%$ SDS buffer. Color intensity was measured at $570 \mathrm{nM}$ in an ELISA plate reader. Absorbance was converted to cell number with reference to a standard curve. For migration studies, the wound healing assay was performed (19). After addition of MSP, RON $\Delta 85$ for 16 or $24 \mathrm{~h}$ dependent on individual cell lines, the area covered by migrated cells was examined under a microscope and photographed.

\section{Results}

Identification of a novel mRNA transcript encoding RON $\Delta 85$. To study RON mRNA transcripts that are not translated but possess regulatory activities upon recombinant expression, total RNAs from colon, breast, and pancreatic cancer cells were subjected to RT-PCR analysis. Three pairs of primers that covers the entire RON extracellular sequence $(-11$ to +1663 , +1311 to +2315 , and +1646 to +2921 ) were used $(23)$. As shown in Table I and Fig. 1A, majority of cDNA fragments were previously reported and coded for the extracellular sequences of RON, RON $\Delta 165$ (deletion of exon 11), RON $\Delta 160$ 
Table I. Identification of various types of cDNA fragments encoding RON or its variants in colon, breast, and pancreatic cancer cells.

\begin{tabular}{|c|c|c|c|c|c|c|}
\hline \multirow[b]{2}{*}{ Cell lines } & \multicolumn{6}{|c|}{ Different types of RON mRNA transcripts ${ }^{a}$} \\
\hline & Wild-type & $\begin{array}{l}\text { Deletion of } \\
\text { exon } 11\end{array}$ & $\begin{array}{c}\text { Deletion of } \\
\text { exons } 5 \text { and } 6\end{array}$ & $\begin{array}{l}\text { Deletion of exons } 5 \text {, } \\
6 \text { and } 11\end{array}$ & $\begin{array}{l}\text { Truncation } \\
\text { at } \mathrm{Met}^{2765}\end{array}$ & $\begin{array}{c}\text { Intron } \\
\text { Insertion }\end{array}$ \\
\hline HT-29 & + & + & + & + & + & - \\
\hline SW620 & + & + & + & - & + & - \\
\hline HCT116 & + & - & - & - & - & - \\
\hline T-47D & + & - & - & - & - & - \\
\hline Du4475 & + & - & + & - & - & + \\
\hline HCC1937 & + & - & - & - & - & - \\
\hline MDA-MB231 & + & + & - & - & + & - \\
\hline MCF-7 & - & - & - & - & - & - \\
\hline BxPC-3 & + & + & - & + & + & - \\
\hline Panc-1 & + & + & - & - & + & - \\
\hline
\end{tabular}

${ }^{a}$ Cell lines expressing mRNA transcripts of RON or its variants are marked as + . Deletion, insertion, and truncation were observed by comparison with published RON gene sequences (23).

(deletion of exons 5 and 6), RON $\Delta 155$ (deletion of exons 5, 6, and 11), and RON $\triangle 52$ (alternatively initiated at $\mathrm{MET}^{913}$ ) (18). Only one novel cDNA fragment $(\sim 1.3 \mathrm{~kb})$ was discovered in Du4475 cells (Fig. 1A and B) (designated as DucDNA-8). To verify these results, Western blot analysis for RON expression was performed. RON variants including RON $\Delta 165$ and RON $\Delta 160$ were detected (Fig. 1C). However, proteins with molecular mass of $80 \mathrm{kDa}$ were not observed. These results were confirmed by immunoprecipitation with $\mathrm{mAb} \mathrm{Zt} / \mathrm{g} 4$ in Du4475 cell lysates followed by Western blot analysis using anti-RON $\alpha$-chain antibody (Fig. 1D). RON, RON $\Delta 165$, and RON $\Delta 160$ were immunoprecipitated from lysates of all four cell lines. However, proteins around $80 \mathrm{kDa}$ were not observed. These result, together with Fig. 1C, demonstrated that DucDNA-8 mRNA is transcribed but not translated in Du4475 cells.

In comparison with RON cDNA sequences, DucDNA-8 had an insertion of 49 nucleotides between the last nucleotide $\mathrm{G}^{1908}$ of the exon 5 and the first nucleotide $\mathrm{A}^{1909}$ of exon 6 . Inserted sequences created six new amino acids followed by a stop codon of TAA (Fig. 1A). Thus, DucDNA-8 encoded a truncated RON variant with a molecular mass of $\sim 85 \mathrm{kDa}$ (RON $\Delta 85$ ) (Fig. 1E), which contains the complete $\alpha$-chain and a portion of extracellular sequences of the $B$-chain. The structure of RON $\Delta 85$ was featured by the complete sema domain followed by a hinge and a portion of the first IPT unit.

Expression and purification of RON $\Delta 85$ from transfected CHO-k1 cells. RON $\Delta 85$ cDNA was constructed, tagged at C-terminus with $\mathrm{V} 5 / \mathrm{His}$, and expressed in CHO-k1 cells. Results in Fig. 2A show that RON $\Delta 85$ existed in cell lysates as a $75 \mathrm{kDa}$ single-chain precursor. In contrast, pro-RON $\Delta 85$ and mature RON $\Delta 85$ (as evident by the presence of the $45 \mathrm{kDa}$ partial B-chain) were detected in the supernatant. The size of mature RON $\Delta 85(\sim 85 \mathrm{kDa})$ was slightly larger than proRON $\Delta 85$, due to glycosylation during the release of RON $\Delta 85$ into the culture fluids. Using a Ni-NTA affinity column for His-tagged protein (Invitrogen), RON $\Delta 85$ was purified from the supernatant (Fig. 2B). Mature RON $\Delta 85$ was bound to the Ni-NTA column in amounts greater than proRON $\Delta 85$. Five micrograms of RON $\Delta 85$ was able to be purified from $150 \mathrm{ml}$ culture supernatant.

Interaction of RON 855 with pro-MSP, MSP, and MSP subunits. To determine if RON $\Delta 85$ interacts with MSP, recombinant pro-MSP, MSP, MSP $\alpha$-, and MSP $\beta$-chain were individually used for co-immunoprecipitation assay followed by Western blot analysis using specific antibodies. Results in Fig. 3A show that pro-MSP or RON $\Delta 85$ was not detected by Western blot analysis in samples of anti-V5 or pro-MSP antibody immunoprecipitation, suggesting that RON $\Delta 85$ did not interact with pro-MSP. However, the formation of the protein complex between RON $\triangle 85$ and MSP was observed (Fig. 3B). In this case, rabbit anti-MSP antibody was able to detect MSP (as evident by the presence of MSP- $\alpha$ chain) in the anti-V5 co-immunoprecipitated samples. This result was further confirmed by Western blotting of RON $\Delta 85$ using anti-MSP antibody in $\mathrm{mAb}$ anti-MSP clone $2 \mathrm{~S}$ co-immunoprecipitated samples. To determine which subunits of MSP were involved in interaction with $\mathrm{RON} \Delta 85$, co-immunoprecipitation was performed where free MSP $\alpha$ - or $\beta$-chain was incubated with RON $\Delta 85$. Results in Fig. $3 \mathrm{C}$ show that the MSP $\alpha$-chain was not detected by Western blotting using anti-MSP $\alpha$-chain antibody after anti-V5 co-immunoprecipitation, indicating that RON $\triangle 85$ does not form a complex with the MSP $\alpha$-chain. However, the MSP $\beta$-chain was detected by Western blotting of anti-V5 co-immunoprecipitated samples using rabbit antibodies to the MSP B-chain (Fig. 3D). These results, together with Fig. $3 \mathrm{~A}-\mathrm{C}$, demonstrate that RON $\Delta 85$ interacts with 
A

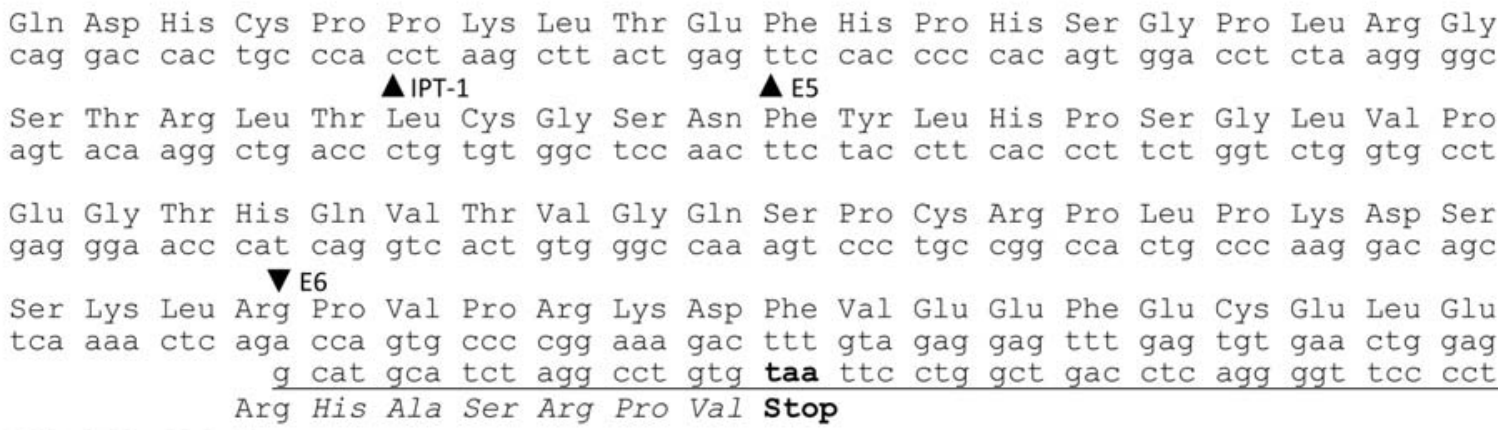

B

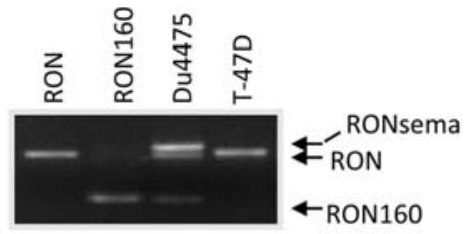

C

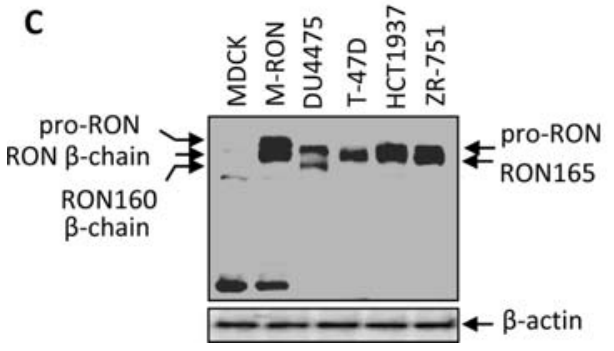

D

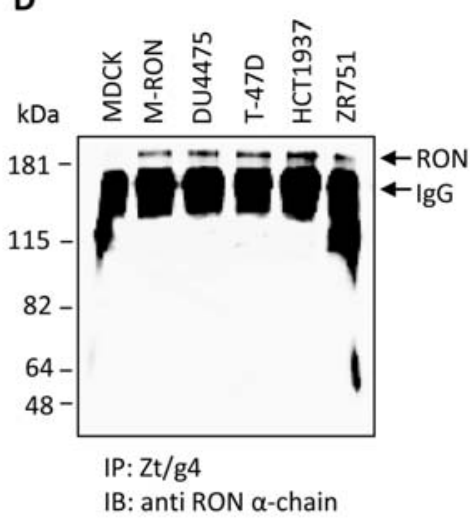

$\mathbf{E}$

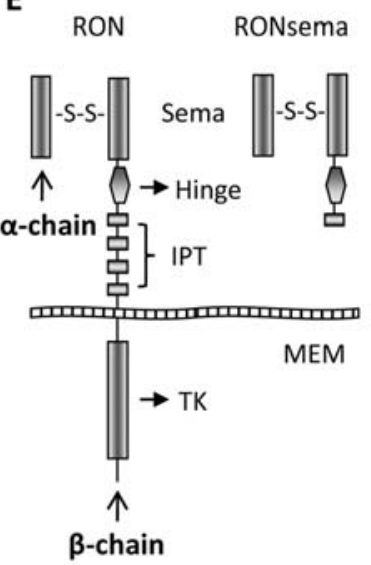

Figure 1. Identification and cloning of the RON $\triangle 85$ mRNA transcript from breast Du4475 cells: (A) Partial sequences of the cDNA fragment encoding RON $\triangle 85$. The beginning of first IPT domain is labeled. The first nucleotide for exon 5 and 6 is indicated with arrow. The inserted 49 nucleotides are underlined. The created stop codon was marked in bold. The sequences of six newly created amino acids are provided. (B) Detection of the cDNA fragment encoding RON $\Delta 85$ by RT-PCR. The RON or RON $\Delta 160$ cDNA was used as the control. The cDNA fragments corresponding to RON, RON $\Delta 160$, or RON $\Delta 85$ were amplified from RNAs derived from Du4475 and T-47D cells. (C) Expression of RON and its variants by breast cancer cells. Cellular proteins (50 $\mu \mathrm{g}$ per sample) were subjected to Western blot analysis using rabbit IgG antibody to RON. MDCK and M-RON cells were used as the control. In Du4475 cells, only pro-RON $\Delta 160$ and RON $\Delta 160$ B-chain were detected. No proteins at molecular mass of $\sim 80 \mathrm{kDa}$ were observed. (D) Validation of RON and its variant expression by breast cancer cells. Cellular proteins ( $300 \mu \mathrm{g}$ per sample) were immunoprecipitated with $\mathrm{mAb} \mathrm{Zt} / \mathrm{g} 4$ followed by Western blot analysis under non-reduced conditions. Mouse mAb to RON $\alpha$-chain was used as the detecting antibody. MDCK and M-RON cells were used as the control. No proteins at molecular mass of $\sim 80 \mathrm{kDa}$ were detected in DU4475 cells. (E) Schematic representation of RON and RON $\Delta 85$. Functional domains in the extracellular sequences including sema, hinge, and IPTs are indicated.

MSP but not pro-MSP. The interaction occurred by binding to the MSP $\beta$-chain.

Effect of RON $\triangle 85$ on MSP-induced RON dimerization and activation. The effect of RON $\triangle 85$ on MSP-induced RON dimerization and activation was studied using M-RON cells as the model. MSP stimulation induced RON dimerization as evident from the appearance of a RON dimer (Fig. 4A). However, the amount of the RON dimer was significantly deceased after RON $\Delta 85$ addition. Judged by band intensities, only $5 \%$ of RON dimers remained. The effect of RON $\Delta 85$ on MSP-induced RON phosphorylation is shown in Fig. 4B. About $90 \%$ of MSP-induced RON phosphorylation was reduced when $35 \mathrm{nM}$ of RON $\Delta 85$ was used. The inhibition was also dose-dependent with progressive reduction of RON phosphorylation in the presence of increasing concentrations of RON $\Delta 85$ (Fig. 4C). Moreover, RON $\Delta 85$ inhibited MSPinduced phosphorylation of downstream signaling proteins (Fig. 4D). More than $50 \%$ reduction of Erk $1 / 2$ and $70 \%$ reduction of $\mathrm{AKT}$ as determined by densitometry analysis were observed. Thus, RON $\Delta 85$ impaired MSP-induced RON dimerization. This impairment affected MSP-induced RON phosphorylation and subsequent activation of downstream signals.

Effect of RON $\Delta 85$ on constitutive activation of RON 160 . RON $\Delta 160$ is a constitutively active variant with oncogenic activities (21). The effect of RON $\Delta 85$ on constitutive phosphorylation was studied using M-RON $\Delta 160$ cells as a model. Results in Fig. 5A show that RON $\Delta 85$ formed a protein complex with RON $\Delta 160$, which was immunoprecipitated using anti-V5 antibody. Such interaction affected spontaneous RON $\Delta 160$ dimerization even in the absence of MSP (Fig. 5B). The effect of RON $\Delta 85$ on constitutive RON $\Delta 160$ phosphorylation is shown in Fig. 5C. RON $\Delta 85$ treatment substantially reduced the levels of RON $\Delta 160$ phosphorylation. MSP had only marginal effect on prevention of RON $\Delta 85$-mediated inhibition. The inhibitory effect of RON $\Delta 85$ on RON $\Delta 160$ 


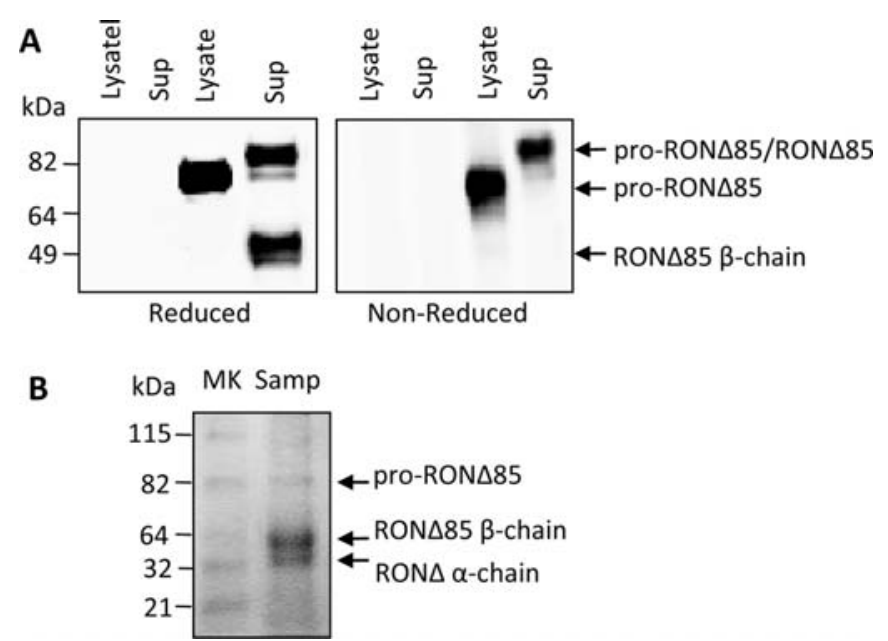

Figure 2. Expression and purification of recombinant RON $\Delta 85$ from cDNA transfected CHO-k1 cells: (A) Cell lysates and culture fluids (50 $\mu$ g protein per sample) from control and transfected $\mathrm{CH}-\mathrm{RON} \Delta 85$ cells were analyzed by Western blot analysis under reduced or non-reduced conditions using anti-V5 antibody. Mature RON $\Delta 85$ was detected only in culture fluids from transfected $\mathrm{CH}-\mathrm{RON} \Delta 85$ cells. (B) Culture fluids $(150 \mathrm{ml})$ of $\mathrm{CH}-\mathrm{RON} \Delta 85$ cells were concentrated and loaded on Ni-NTA column. This column was much better in purification of mature RON $\Delta 85$ than pro-RON $\Delta 85$. The obtained RON $\Delta 85$ ( $\sim 2 \mu \mathrm{g}$ protein/sample) was analyzed in a $10 \%$ SDS-PAGE, stained with Coomassie blue, and photographed. Both RON $\Delta 85 \alpha$ - and $\beta$-chain were observed. The amount of pro-RON $\Delta 85$ was less than $10 \%$ of the total preparation. MK, molecular marker.

phosphorylation was dose-dependent. Maximal inhibition was seen when $35 \mathrm{nM}$ of RON $\Delta 85$ was used. In addition, we observed the inhibitory effect of RON $\Delta 85$ on constitutive phosphorylation of Erk1/2 and AKT in M-RON $\Delta 160$ cells (Fig. 5D). Thus, results in Fig. 5 demonstrate that RON $\Delta 85$ directly interacts with RON $\Delta 160$. Such interactions impair spontaneous RON $\Delta 160$ dimerization, which leads to diminished tyrosine phosphorylation and attenuated downstream signaling activities.

Effect of RON $\triangle 85$ on MSP-RON signaling pathways and its specificity. Four cancer cell lines (SW837, HCT116, T-47D, BxPC-3) were used to validate the results described above. RON $\Delta 85$ treatment significantly inhibited MSP-induced RON phosphorylation in all four cell lines tested (Fig. 6A). The levels of inhibition varied among cell lines and ranged from 50 to $90 \%$. The inhibitory effect of RON $\Delta 85$ on phosphorylation of Erk1/2 and AKT was also confirmed using BxPC-3 and HCT116 cells as model (Fig. 6B). More than 50\% of reduction in MSP-dependent or independent Erk1/2 phosphorylation was observed in both cell lines. Significant inhibition by RON $\Delta 85$ on constitutive AKT phosphorylation was also observed (Fig. 6B). Taken together, these results suggest that RON $\Delta 85$ inhibited phosphorylation of RON, Erk1/2 and AKT in cancer cells.

Cross-talk between RON and MET or EGFR exists in cancer cells $(24,25)$. We selected BxPC-3 cells as the model since they express RON, MET and EGFR. Results in Fig. 6C show that HGF-induced MET phosphorylation was not affected by the presence of RON $\Delta 85$. Similarly, EGF-induced EGFR phosphorylation was not affected by RON $\Delta 85$ (Fig. 6C). In both cases, levels of phosphorylation were comparable to those of positive controls. These results suggest that the effect of RON $\triangle 85$ is specific only to RON and not to structure-related MET or unrelated EGFR.
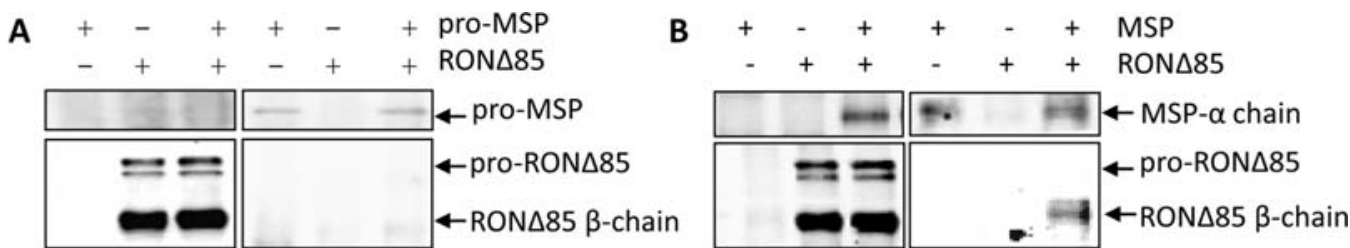

IP: Mouse anti-v5 (left) or 2S (right); IB: Rabbit anti-MSP (up) or V5 (down)

C

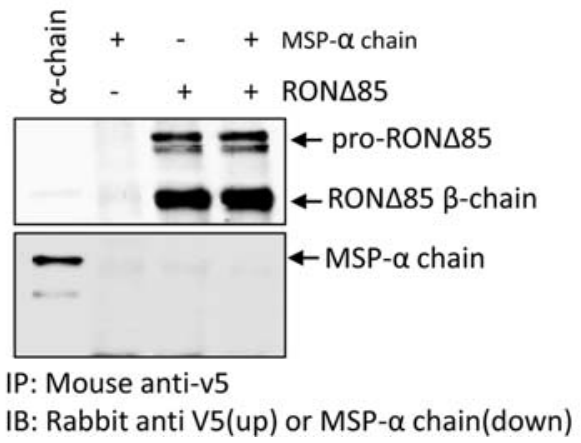

D

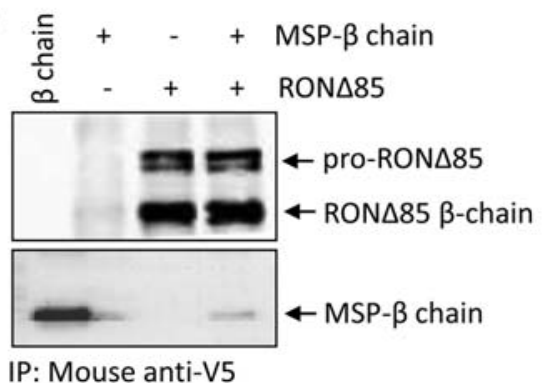

use anti-V5

IB: Rabbit anti V5(up) or MSP- $\beta$ chain(down)

Figure 3. Direct interaction of RON $\Delta 85$ with MSP and its $\beta$-chain: (A) RON $\Delta 85$ did not interact with pro-MSP. Purified RON $\Delta 85$ (2 $\mu$ g/sample) was mixed with or without recombinant human pro-MSP $\left(1.5 \mu \mathrm{g}\right.$ per sample) at $4^{\circ} \mathrm{C}$ for $12 \mathrm{~h}$ in PBS. Samples were then immunoprecipitated with mouse mAb to V5 or to pro-MSP (clone 2S) followed by Western blot analysis using rabbit antibodies to V5 or to MSP. (B) RON $\Delta 85$ interacted with MSP. Experimental conditions were similar to those in A. MSP was mixed with RON $\Delta 85$ followed by anti-V5 or anti-MSP immunoprecipitation. (C) RON $\Delta 85$ failed to interact with the MSP $\alpha$-chain. Purified RON $\Delta 85(2 \mu \mathrm{g} / \mathrm{sample})$ were mixed in PBS with or without recombinant human MSP- $\alpha$ chain $\left(2.5 \mu \mathrm{g}\right.$ per sample) at $4{ }^{\circ} \mathrm{C}$ for $12 \mathrm{~h}$. Proteins were immunoprecipitated using mouse antibody to V5 followed by Western blot analysis using rabbit anti-V5 or anti-MSP- $\alpha$ chain antibodies (38). The pure MSP- $\alpha$ chain was used as the control. (D) RON $\Delta 85$ interacted with the MSP- $\beta$ chain. Experimental conditions were similar to those in C. The MSP- $\beta$ chain was used at $2.5 \mu \mathrm{g} /$ sample. After anti-V5 immunoprecipitation, the presence of the MSP- $\beta$ chain was determined by Western blot analysis using rabbit antibody to V5 or to the MSP-ß chain (38). 

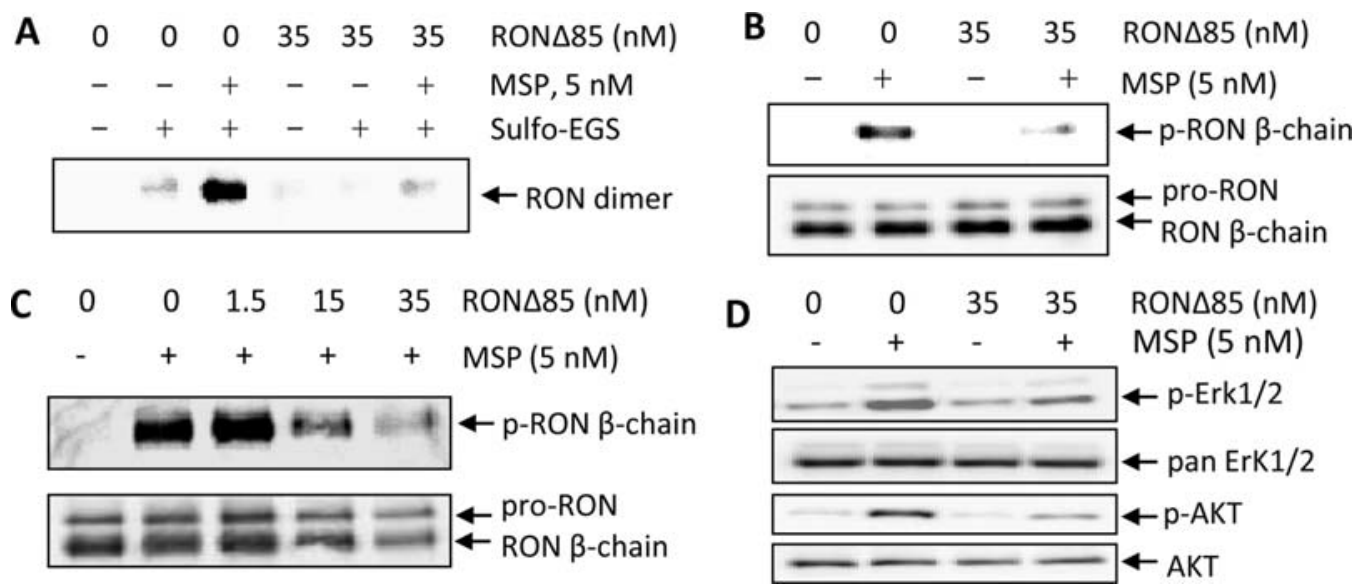

Figure 4. Inhibitory effect of RON $\triangle 85$ on MSP-induced phosphorylation of RON and downstream signaling proteins: M-RON cells were used in all experiments. (A) RON $\triangle 85$ inhibits MSP-induced RON dimerization. Cells $\left(2 \times 10^{6}\right.$ cells in $60 \mathrm{~mm}$ culture dish) were stimulated at $37^{\circ} \mathrm{C}$ for 30 min with or without $5 \mathrm{nM}$ of MSP in the presence or absence of $35 \mathrm{nM}$ of purified RON $\triangle 85$ followed by addition of the chemical cross-linker Sulfo-EGS. The formation of the RON dimer was determined by Western blot analysis using antibodies to RON. (B) RON $\triangle 85$ inhibits MSP-induced RON phosphorylation. After MSP stimulation for $15 \mathrm{~min}$ in the presence or absence of RON $\Delta 85(35 \mathrm{nM}), \mathrm{Zt} / \mathrm{g} 4$ immunoprecipitation was performed followed by Western blot analysis using mAb PY-100 for phosphorylated RON. The membrane was also reprobed with antibody to RON as the loading control. (C) Dose-dependent inhibition by RON $\triangle 85$ of RON phosphorylation. Experimental conditions were similar to those in B, except for difference in amount of RON $\Delta 85$ added to the cell culture. (D) Effect of RON $\triangle 85$ on RON-mediated phosphorylation of Erk1/2 and AKT. Cells stimulation and Western blot analysis were similar to those in B. Phosphorylated Erk1/2 and AKT were detected by Western blot analysis using individual antibodies. Data shown here are from one of three experiments with similar results.
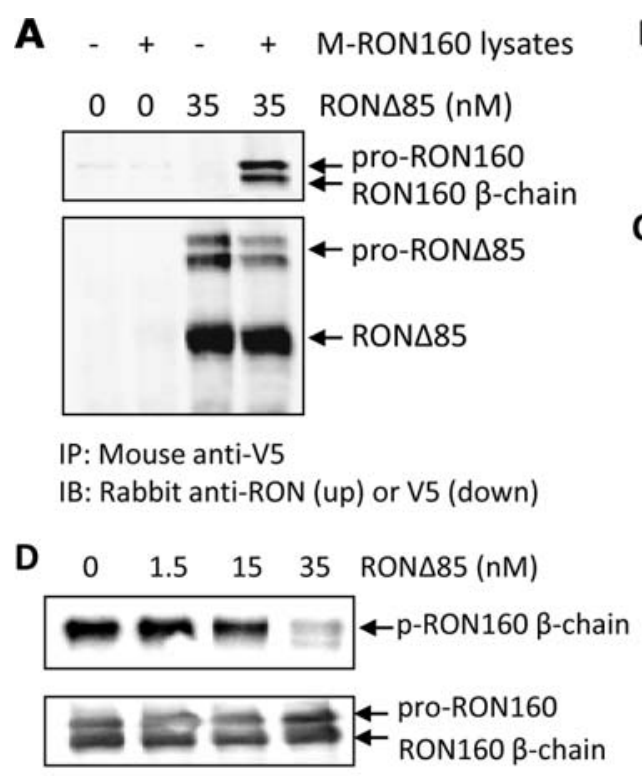
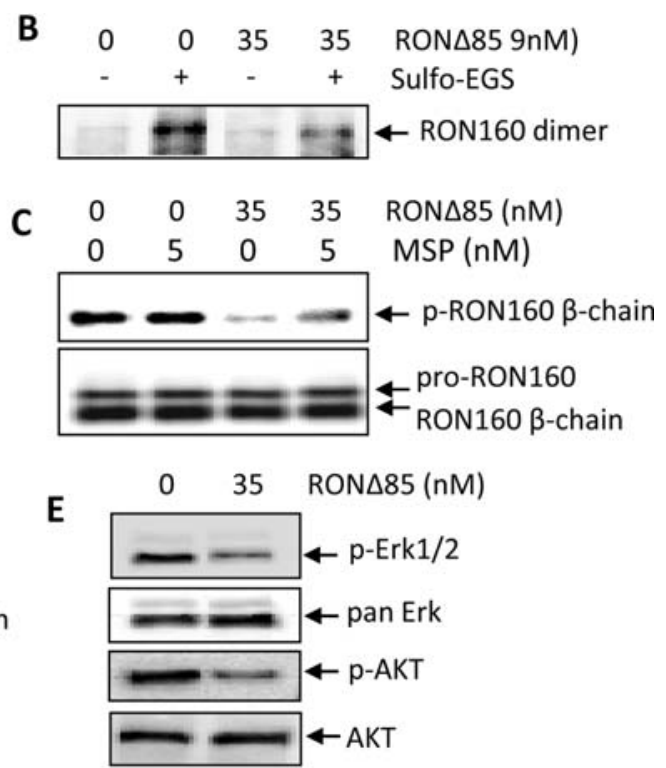

Figure 5. Inhibitory effect of RON $\Delta 85$ on MSP-independent RON phosphorylation and downstream signaling activation. M-RON $\Delta 160$ cells were used as the model in all experiments. (A) Interaction of RON $\Delta 85$ with RON $\Delta 160$. Proteins $(300 \mu \mathrm{g} / \mathrm{sample})$ from cell lysates were mixed with or without RON $\Delta 85$ $(35 \mathrm{nM})$ at $4^{\circ} \mathrm{C}$ for $12 \mathrm{~h}$ followed by anti-V5 immunoprecipitation. The presence of RON $\Delta 160$ or RON $\Delta 85$ was determined by Western blot analysis using rabbit anti-RON or V5 antibody, respectively. (B) Effect of RON $\Delta 85$ on RON $\Delta 160$ dimerization. Experiments were performed as detailed in Fig. 4 A except that MSP is not included. The RON $\Delta 160$ dimer was determined by Western blot analysis using rabbit anti-RON antibodies. (C) Effect of RON $\Delta 85$ on constitutive RON $\Delta 160$ phosphorylation. Cell stimulation, RON $\Delta 85$ treatment, and Western blot analysis were done as described in Fig. $4 \mathrm{~B}$. Protein intensity was determined by densitometric analysis. (D) Dose-dependent inhibition by RON $\Delta 85$ of constitutive RON $\Delta 160$ phosphorylation. Experiments were performed as described in Fig. 4C. MSP was not included. (E) Effect of RON $\Delta 85$ on constitutive Erk1/2 and AKT phosphorylation. Cells were treated with RON $\Delta 85$ followed by Western blot analysis as described in Fig. 4D. Data shown here are from one of three experiments with similar results.

Effect of RON $\triangle 85$ on MSP-dependent or independent growth of cancer cells. MSP-dependent and independent cell growth was first studied. M-RON and M-RON $\Delta 160$ cells were used as the model. Results in Fig. 7A show that RON $\Delta 85$ moderately inhibited MSP-induced cell proliferation. This effect was also seen in M-RON $\Delta 160$ cells, in which cell proliferation is independent of MSP stimulation. The inhibition was validated in BxPC-3 and HCT116 cells (Fig. 7B). Again, only moderate inhibition was observed. The rate of inhibition was about $60 \%$ for BxPC-3 and $36 \%$ for HCT 116. 


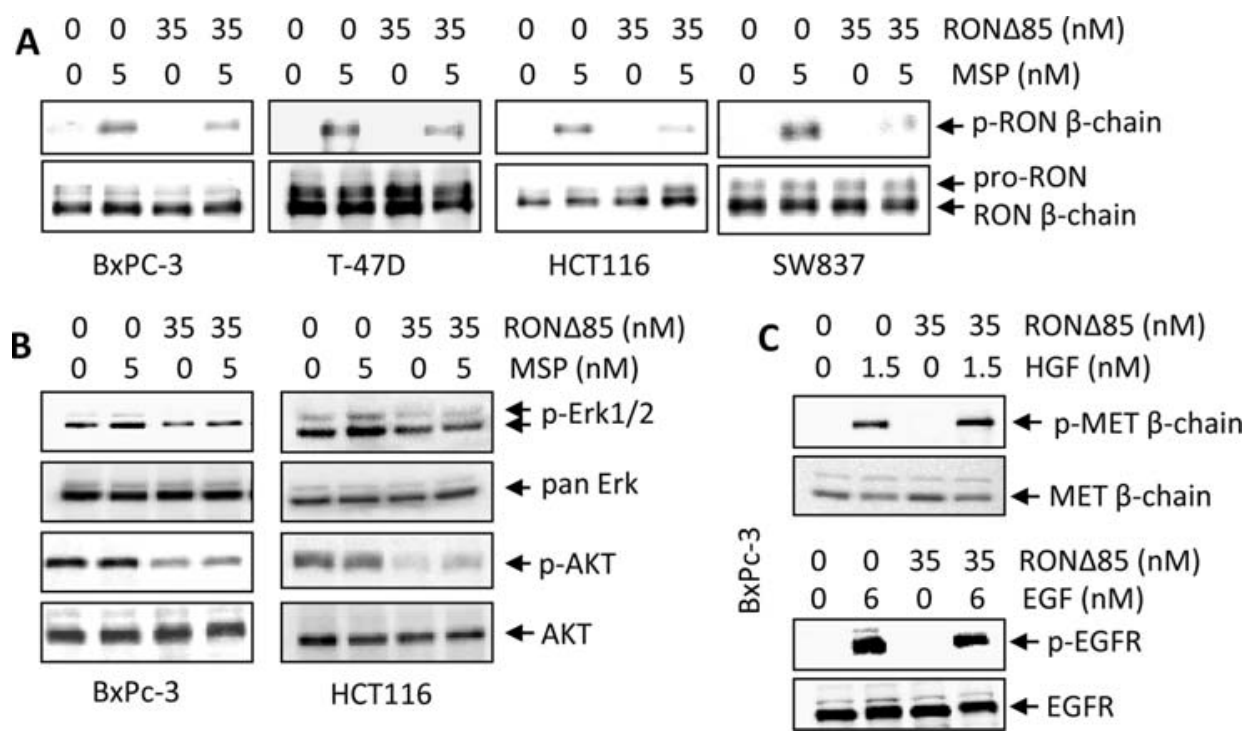

Figure 6. Effect of RON $\Delta 85$ on phosphorylation of RON, MET, EGFR, and downstream signaling proteins. (A) Inhibitory effect of RON $\Delta 85$ on RON phosphorylation in four cancer cell lines. Cells $\left(3 \times 10^{6}\right.$ cells per sample) were stimulated at $37^{\circ} \mathrm{C}$ for 15 min by MSP in the presence or absence of RON $\Delta 85$. After Zt/g4 immunoprecipitation of cellular proteins, phosphorylation of RON was detected by Western blot analysis using mAb PY-100. Membranes were also reprobed with rabbit antibodies to RON as the loading control. (B) Effect of RON $\Delta 85$ on Erk1/2 and AKT phosphorylation. BxPC-3 and HCT116 cells were used as the model. Experimental procedures were similar to those in Fig. 4D. Protein intensity was determined by densitometric analysis. (C) Effect of RON $\triangle 85$ on phosphorylation of MET or EGFR. BxPC-3 cells were stimulated with HGF or EGF for 15 min followed by immunoprecipitation using corresponding antibodies. MET and EGFR phosphorylation was determined by Western blot analysis using PY-100. Membranes were also reprobed for MET and EGFR using corresponding antibodies, respectively, as the loading controls.
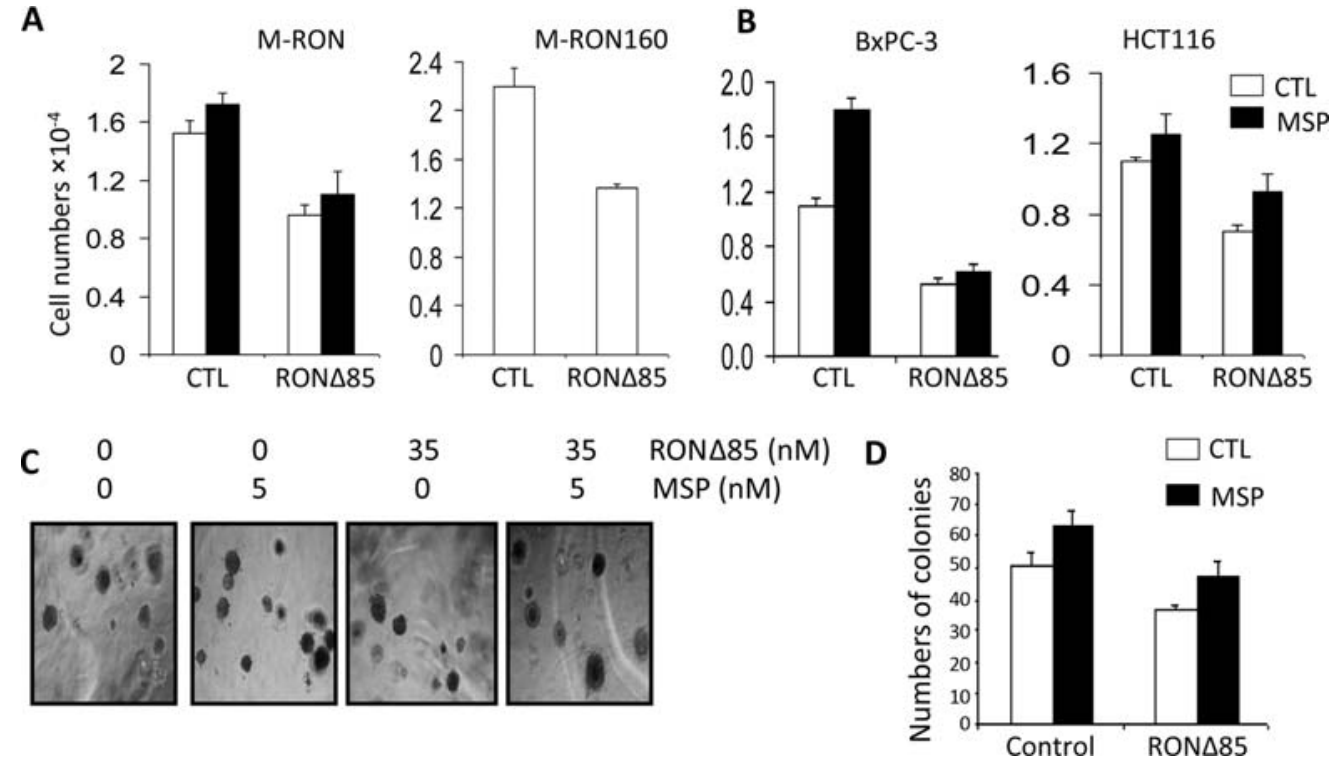

Figure 7. Effect of RON $\Delta 85$ on MSP-induced cell growth and colony formation: (A) Cells at $1 \times 10^{4}$ cells/well in triplicate in a 96 -well plate were cultured with or without MSP, RON $\Delta 85$, or their combination for 5 days. Cells were stained as previously described (19). Cell number was determined by comparison with standard curve. (B and C) HCT116 cells at $2 \times 10^{3}$ cells/well in soft agar in duplicate were cultured for 18 days. Colonies in individual wells were photographed and counted.

The effect of RON $\Delta 85$ on anchorage-independent growth of HCT116 cells were also determined (Fig. 7C and D). Number of both spontaneous and MSP-stimulated colonies was moderately reduced in the presence of RON $\Delta 85$. Similar results were also obtained when BxPC-3 cells were used. Thus, results in Fig. 7 demonstrate that RON $\Delta 85$ moderately inhibited MSP-dependent or independent cell growth of cancer cells.
Effect of RON $\Delta 85$ on MSP-dependent or -independent cell migration. Migration of BxPC-3 and HCT116 cells was assessed by the wound healing assay. Results in Fig. 8A show that BxPC-3 cells had relatively high levels of spontaneous migration, which was enhanced further by MSP stimulation. RON $\Delta 85$ inhibited both spontaneous and MSP-induced cell migration. The percentage of space covered by migrated cells was reduced from 47 to $28 \%$ in unstimulated cells and 


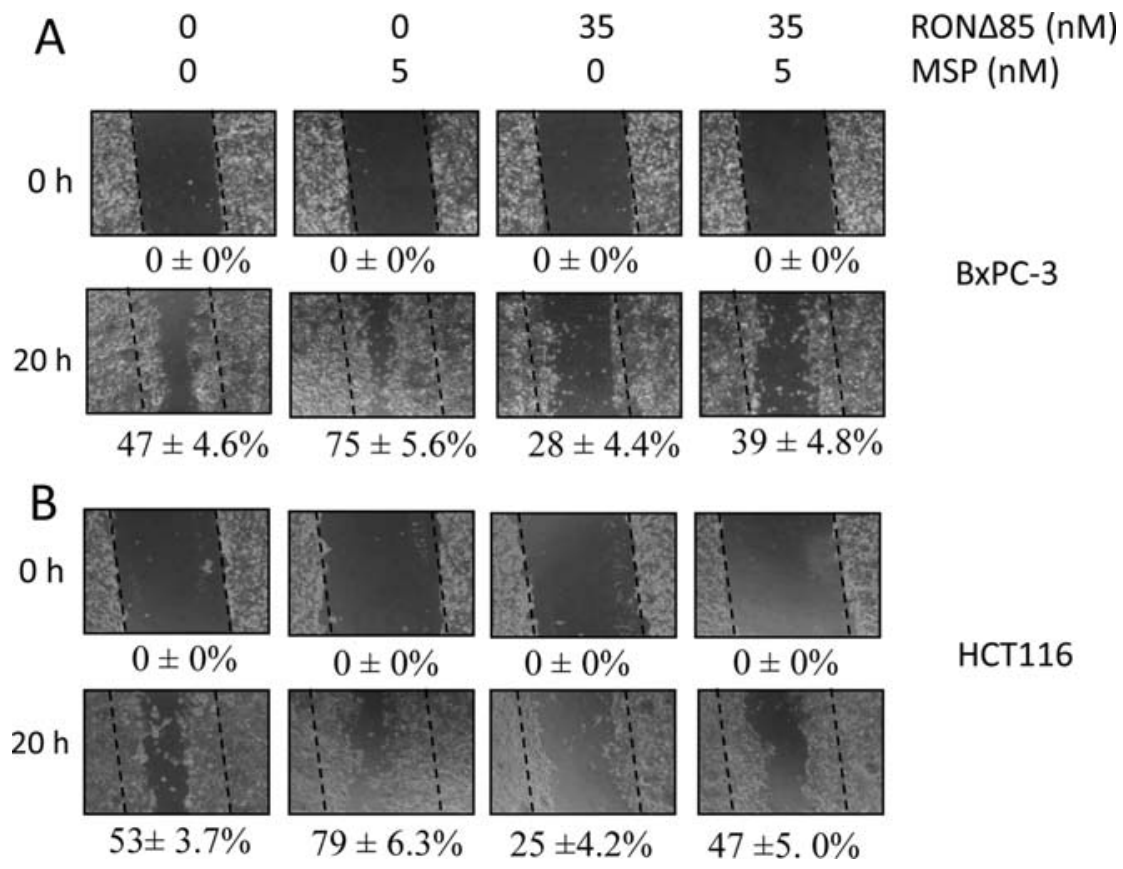

Figure 8. Effect of RON $\Delta 85$ on spontaneous or MSP-induced cell migration: The wound healing assay was performed as previously described (19). (A) BxPC-3 and (B) HCT116 cells were used as the model. The wound closure was calculated as percentages of the area covered by the migrated cells.

75 to $39 \%$ in MSP-stimulated cells, respectively. Similar patterns of inhibition were also observed in HCT116 cells (Fig. 8B). The percentage of space covered by migrated cells was reduced from 53 to $25 \%$ in MSP un-treated cells and 79 to $47 \%$ in MSP treated cells, respectively. These results suggest that RON $\Delta 85$ inhibited cell migration in MSPdependent or -independent manner.

\section{Discussion}

The central findings of this study are the discovery of RON $\Delta 85$ and its inhibitory activities in the MSP-RON pathway in epithelial cancer cells. As indicated in Fig. 1, RON $\Delta 85$ mRNA transcript was not translated in cancer cells but displayed inhibitory activities when used as a recombinant protein. Previous studies have shown that aberration in the MSP-RON pathway contributes to tumorigenic progression in cancer cells (1). Disruption of MSP-RON signaling by RON specific mAbs (8) and small molecular inhibitors $(10,26)$ inhibits tumorigenic activities in vitro and impairs tumor growth in vivo. These activities are mainly mediated by interaction of mAbs or agents with functional domains in the RON extracellular sequences or in the kinase domain, which impairs RON dimerization, phosphorylation, kinase activities, and subsequently inactivates downstream signaling events $(10,26)$. Consistent with these observations, the results from this study demonstrate that RON $\Delta 85$ mRNA transcript exists in cancer cells. Upon expression as a recombinant protein, RON $\Delta 85$ acts as an antagonist to RON-mediated signaling and tumorigenic activities.

Altered RON expression exists in primary cancer samples and established cell lines (3), which is characterized by the presence of various mRNA transcripts and corresponding variants (18). A survey by PCR techniques of primary cancer samples has shown that various RON mRNA transcripts such as those from RON165, RON $\Delta 160$, and RON155 were wildly produced by different types of cancer cells (27). A recent study further revealed that different mRNA transcripts encoding known or unknown RON variants exist in human glioma samples with relatively high frequencies (28). Currently, seven RON variants have been identified and their corresponding mRNA transcripts have been cloned (18). These RON mRNA transcripts are formed mainly by aberrant splicing, insertion, alternative initiation, protein truncation, and other unknown mechanisms (18). Thus, the RON gene transcription is altered in cancer cells. Different lengths of RON mRNA transcripts are synthesized in tumor cells, which increase the complexity and diversity of the RON receptor.

Cellular translation of mRNA transcripts is a regulated process. Only certain transcripts encoding RON, RON $\Delta 165$, RON $\Delta 160$, and RON $\Delta 155$ or others are translated into proteins (18). Majority of the transcripts are degraded quickly without protein synthesis. Currently, mechanism(s) underlying the generation of various RON transcripts in cancer cells are largely unknown. Also, mechanism(s) that governs the translation of a particular RON mRNA transcript are also unknown. Nevertheless, the existence of these untranslated transcripts provides opportunities to study if they possess regulatory activities after recombinant expression. Unique RTK variants derived from cancer cells have been shown to have therapeutic effect in various tumor models (29-31). Thus, identification of RON $\Delta 85$ with antagonistic activities opens an avenue for the development of potential therapeutics for targeted cancer therapy.

Limited surveys have revealed that Du4475 cell line is the only one that harbors RON $\Delta 85$ mRNA transcript. This suggests that the frequency of this particular transcript is low and its formation may occur through a random process. 
Nevertheless, the existence of such transcript with an insertion of 49 nucleotides seems not to be a normal event. A similar transcript, almost identical to the RON $\Delta 85$ mRNA, has recently been identified (28). The transcript is generated by alternative splicing that deletes exon 6 . This deletion causes a reading-frame shift, which creates a stop codon after addition of three amino acids. The synthesized protein RON $\Delta 90$ inhibits MSP-induced RON phosphorylation and cell migration. Sequence comparison reveals that RON $\Delta 90$ differs from RON $\Delta 85$ only in last six amino acids. Unlike RON $\Delta 85$ mRNA found only in a single cell line, RON $\triangle 90$ mRNA has been detected in three normal brain tissues and in the majority of brain tumor samples (13/17 cases). It was also produced by colon cancer DLD- 1 cells. Moreover, RON $\Delta 90$ was detected in culture supernatants from 5 glioblastoma cell lines (28). It appears that RON $\Delta 90$ mRNA transcript is wildly expressed, particularly in normal and cancerous cells of brain. In contrast, RON $\Delta 85$ mRNA expression is limited to a particular cell line only.

Functional domains in the RON extracellular sequence including sema and IPT domains are important in ligand recognition and subsequent activation (7). MSP utilizes its high affinity binding site located on the $\beta$-chain to bind RON (12). However, the ligand-recognition pocket on the RON molecular is still largely unknown. To address the importance of the sema domain in the MSP-RON pathway, two studies have been conducted. One study showed that the soluble sema domain of RON, produced by recombinant techniques, impairs MSP binding to RON, and inhibits MSPinduced RON phosphorylation and cellular responsiveness (17). Although direct interaction has not been proved, results provide an important clue indicating that the sema domain participates in ligand binding by the full-length receptor. A recent study provided additional evidence showing that the sema domain plays a role in MSP-induced RON activation (28). Studies in MET, the homologue of RON, have shown that the sema domain in the MET receptor contains a $\mathrm{HGF} /$ SF-interaction pocket (32). Therefore, the sema domain of RON molecule possesses a similar function. Data from this study extend the previous findings and provide a direct evidence showing that the sema domain of RON physically forms a protein complex with MSP, which is mediated by binding to the MSP $\beta$-chain. Since the MSP B-chain contains a high affinity binding site, it is reasoned that the corresponding ligand-recognition pocket may reside in the sema domain of RON.

Study of RON $\Delta 85$ has revealed the following biochemical features: First, RON $\Delta 85$ directly interacts with MSP and the MSP $\beta$-chain but not with pro-MSP or the MSP $\alpha$-chain (Fig 3). This was confirmed by co-immunoprecipitation in which MSP and RON $\Delta 85$ was detected by Western blot analysis using their corresponding antibody, respectively. These results indicate that the sema domain contains a ligand-recognition pocket(s). Second, RON $\Delta 85$ inhibits MSPinduced RON dimerization and phosphorylation (Fig. 4). Both MSP-induced RON dimerization and subsequent phosphorylation were significantly diminished upon RON $\Delta 85$ treatment. These results indicate that RON $\Delta 85$ is an effective antagonist to MSP-induced RON activation. Third, RON $\Delta 85$ impairs MSP-induced phosphorylation of downstream signaling molecules (Fig. 4D). The inhibition by RON $\Delta 85$ of MSP-induced Erk1/2 and AKT phosphorylation indicates that RON $\triangle 85$ blocks the signal transduction from RON to downstream molecules. Fourth, RON $\Delta 85$ physically forms a complex with RON $\Delta 160$, inhibits its dimerization, reduces spontaneous phosphorylation, and attenuates downstream signaling activation (Fig. 5). The formation of RON $\Delta 85$ RON $\Delta 160$ complex affects spontaneous RON $\Delta 160$ dimerization, which impairs constitutive phosphorylation of RON $\Delta 160$, Erk $1 / 2$ and AKT. It is reasoned that the semasema interaction between RON $\Delta 85$ and RON $\Delta 160$ is the possible mechanism underlying the observed effect $(17,29)$. Moreover, the negative effect on RON $\Delta 160$ indicates that RON $\Delta 85$ regulates MSP-independent RON activation. Fifth, the inhibitory effect of RON $\Delta 85$ acts not only in transfected cells but also in established cancer cell lines (Fig. 6). MSPinduced phosphorylation of RON, Erk1/2 and AKT in cancer cells was inhibited after RON $\Delta 85$ treatment. Finally, the effect of RON $\triangle 85$ is specific to RON and not to structure-related MET or unrelated EGFR, even though both are known to have cross-talk with RON. In summary, results from Figs. 3 to 6 demonstrate that RON $\Delta 85$ is a specific antagonist to RONmediated signaling events. They also serve as a mechanistic mode of RON $\triangle 85$ in inhibition of the MSP-RON pathway.

Activation of the MSP-RON pathway directs a cellular program known as invasive growth characterized by increased cell replication, migration, and matrix invasion (33). Results from studying RON $\Delta 85$ in regulating tumorigenic activities revealed that RON $\triangle 85$ inhibits cell growth and colony formation in both transfected and established tumor cell lines. Both MSP-dependent and -independent cell growth were affected. These results indicate that the inhibitory effect on signaling events has biological consequences. However, the effectiveness of RON $\Delta 85$ on cell growth varied among cell lines. Significant inhibition was observed only in BxPC-3 cells in contrast to moderate inhibition in HCT116 cells. Moderate levels of inhibition were also seen in anchorage-independent growth of HCT116 cells in soft agar. Such differences in inhibition may reflect the differential dependency of cancer cells on RON-mediated signaling. The relatively weak inhibition by RON $\Delta 85$ on Erk1/2 phosphorylation could also be a reason for the modest inhibition. Regardless of the mechanisms underlying RON $\triangle 85$-mediated growth inhibition, results in Fig. 7 imply that RON $\Delta 85$ has the ability to inhibit cell growth and its effectiveness is dependent on cellular contexts of individual cell lines.

The effect of RON $\Delta 85$ on MSP-dependent or -independent cell migration is strong as compared to cell growth. More than $50 \%$ of inhibition (67\% for BxPC-3 cells and $53 \%$ for HCT116 cells, respectively) was achieved in MSP-independent cell migration. Furthermore, inhibition of MSP-dependent migration was also achieved around $67 \%$ for BxPC-3 cell and $41 \%$ for HCT116 cells. Strong inhibition seems to be linked to the effect of RON $\Delta 85$ on AKT phosphorylation. RON $\Delta 85$ substantially inhibited spontaneous and MSPinduced ATK phosphorylation in both colon and pancreatic cell lines tested (Fig. 6). Activation of the PI-3 kinase-AKT pathway is involved in RON-mediated cell migration and matrix invasion (34-36). Recent studies further demonstrate that PI-3 kinase activation and AKT phosphorylation were 
dramatically reduced upon siRNA-mediated silencing of the RON gene expression (37). These negative effects significantly affect in vitro cell migration, invasion and in vivo tumor metastasis (37). Considering these results, it is likely that RON $\Delta 85$-induced inhibition of AKT phosphorylation is a mechanism responsible for the inhibition of cell migration. Definitely, other pathways are also involved in inhibition of cell migration. Further studies should provide insight into the mechanisms by which RON $\Delta 85$ regulates RON signaling and its biological activities.

\section{Acknowledgements}

We thank Ms. Snehal S. Padhye (School of Pharmacy at TTUHSC, Amarillo, TX) for editing the manuscript. This study was supported in part by NIH R01 grant (CA91980) and Amarillo Area Foundation (MHW). The authors declare no conflict interest in this study.

\section{References}

1. Wagh PK, Peace BE and Waltz SE: Met-related receptor tyrosine kinase Ron in tumor growth and metastasis. Adv Cancer Res 100: 1-33, 2008.

2. Camp ER, Liu W, Fan F, Yang A, Somcio R and Ellis LM: $\mathrm{RON}$, a tyrosine kinase receptor involved in tumor progression and metastasis. Ann Surg Oncol 12: 273-281, 2005.

3. Wang MH, Lee W, Luo YL, Weis MT and Yao HP: Altered expression of the RON receptor tyrosine kinase in various epithelial cancers and its contribution to tumourigenic phenotypes in thyroid cancer cells. J Pathol 213: 402-411, 2007.

4. Welm AL, Sneddon JB, Taylor C, Nuyten DS, van de Vijver MJ, Hasegawa $\mathrm{BH}$ and Bishio JM: The macrophage-stimulating protein pathway promotes metastasis in a mouse model for breast cancer and predicts poor prognosis in humans. Proc Natl Acad Sci USA 104: 7570-7575, 2007

5. Zinser GM, Leonis MA, Toney K, Pathrose P, Thobe M, Kader SA, Peace BE, Beauman SR, Collins MH and Waltz SE: Mammary-specific Ron receptor overexpression induces highly metastatic mammary tumors associated with beta-catenin activation. Cancer Res 66: 11967-11974, 2006.

6. Chen YQ, Zhou YQ, Fu LH, Wang D and Wang MH: Multiple pulmonary adenomas in the lung of transgenic mice overexpressing the RON receptor tyrosine kinase. Recepteur d' origine nantais. Carcinogenesis 23: 1811-1819, 2002.

7. Wang MH, Yao HP and Zhou YQ: Oncogenesis of RON receptor tyrosine kinase: a molecular target for malignant epithelial cancers. Acta Pharmacol Sin 27: 641-650, 2006.

8. O'Toole JM, Rabenau KE, Burns K, Lu D, Mangalampalli V, Balderes P, Covino N, Bassi R, Prewett M, Gottfredsen KJ, Thobe MN, Cheng Y, Li Y, Hicklin DJ, Zhu Z, Waltz SE, Hayman MJ, Ludwig DL and Pereira DS: Therapeutic implications of a human neutralizing antibody to the macrophagestimulating protein receptor tyrosine kinase $(\mathrm{RON})$, a c-MET family member. Cancer Res 66: 9162-9170, 2006.

9. Xu XM, Wang D, Shen Q, Chen YQ and Wang MH: RNAmediated gene silencing of the RON receptor tyrosine kinase alters oncogenic phenotypes of human colorectal carcinoma cells. Oncogene 23: 8464-8474, 2004.

10. Zhang Y, Kaplan-Lefko PJ, Rex K, Yang Y, Moriguchi J, Osgood T, Mattson B, Coxon A, Reese M, Kim TS, Lin J, Chen A, Burgess TL and Dussault I: Identification of a novel recepteur d'origine nantais/c-met small-molecule kinase inhibitor with antitumor activity in vivo. Cancer Res 68: 6680-6687, 2008.

11. Dussault I and Bellon SF: From concept to reality: the long road to c-Met and RON receptor tyrosine kinase inhibitors for the treatment of cancer. Anticancer Agents Med Chem 9: 221-229, 2009.

12. Wang MH, Julian FM, Breathnach R, Godowski PJ, Takehara T, Yoshikawa W, Hagiya M and Leonard EJ: Macrophage stimulating protein (MSP) binds to its receptor via the MSP beta chain. J Biol Chem 272: 16999-167004, 1997.
13. Danilkovitch A, Miller M and Leonard EJ: Interaction of macrophage-stimulating protein with its receptor. Residues critical for beta chain binding and evidence for independent alpha chain binding. J Biol Chem 274: 29937-29943, 1999.

14. Ronsin C, Muscatelli F, Mattei MG and Breathnach R: A novel putative receptor protein tyrosine kinase of the met family. Oncogene 8: 1195-1202, 1993.

15. Stamos J, Lazarus RA, Yao X, Kirchhofer D and Wiesmann C: Crystal structure of the HGF beta-chain in complex with the Sema domain of the Met receptor. EMBO J 23: 2325-2335, 2004.

16. Basilico C, Arnesano A, Galluzzo M, Comoglio PM and Michieli P: A high affinity hepatocyte growth factor-binding site in the immunoglobulin-like region of Met. J Biol Chem 283: 21267-21277, 2008.

17. Angeloni D, Danilkovitch-Miagkova A, Miagkov A, Leonard EJ and Lerman MI: The soluble sema domain of the RON receptor inhibits macrophage-stimulating protein-induced receptor activation. J Biol Chem 279: 3726-3732, 2006.

18. Lu Y, Yao HP and Wang MH: Multiple variants of the RON receptor tyrosine kinase: biochemical properties, tumorigenic activities, and potential drug targets. Cancer Lett 257: 157-164, 2007.

19. Zhang K, Yao HP and Wang MH: Activation of RON differentially regulates claudin expression and localization: role of claudin-1 in RON-mediated epithelial cell motility. Carcinogenesis 29: 552-559, 2008.

20. Yao HP, Luo YL, Feng L, Cheng LF, Lu Y, Li W and Wang MH: Agonistic monoclonal antibodies potentiate tumorigenic and invasive activities of splicing variant of the RON receptor tyrosine kinase. Cancer Biol Ther 5: 1179-1186, 2006.

21. Zhou YQ, He C, Chen YQ, Wang D and Wang MH: Altered expression of the RON receptor tyrosine kinase in primary human colorectal adenocarcinomas: Generation of different splicing variants and their oncogenic potential. Oncogene 22: 186-197, 2003.

22. Wang MH, Lao WF, Wang D, Luo YL and Yao HP: Blocking tumorigenic activities of colorectal cancer cells by a splicing $\mathrm{RON}$ receptor variant defective in the tyrosine kinase domain. Cancer Biol Ther 6: 1121-1129, 2007.

23. Angeloni D, Danilkovitch-Miagkova A, Ivanov SV, Breathnach R, Johnson BE, Leonard EJ and Lerman MI: Gene structure of the human receptor tyrosine kinase RON and mutation analysis in lung cancer samples. Genes Chromosomes Cancer 29: 147-156, 2000.

24. Follenzi A, Bakovic S, Gual P, Stella MC, Longati P and Comoglio PM: Cross-talk between the proto-oncogenes Met and Ron. Oncogene 19: 3041-3049, 2000.

25. Peace BE, Hill KJ, Degen SJ and Waltz SE: Cross-talk between the receptor tyrosine kinases Ron and epidermal growth factor receptor. Exp Cell Res 289: 317-325, 2003.

26. Christensen JG, Schreck R, Burrows J, Kuruganti P, Chan E, Le P, Chen J, Wang X, Ruslim L, Blake R, Lipson KE, Ramphal J, Do S, Cui JJ, Cherrington JM and Mendel DB: A selective small molecule inhibitor of c-Met kinase inhibits cMet-dependent phenotypes in vitro and exhibits cytoreductive antitumor activity in vivo. Cancer Res 63: 7345-7355, 2003.

27. Wortinger M and Liu L: RON splice variant prevalence in human tumors. Proc 99th AACR Annual meeting. Abst. 3610, 2008.

28. Eckerich C, Schulte A, Martens T, Zapf S, Westphal M and Lamszus K: RON receptor tyrosine kinase in human gliomas: expression, function, and identification of a novel soluble splice variant. J Neurochem 109: 969-980, 2009.

29. Michieli P, Mazzone M, Basilico C, Cavassa S, Sottile A, Naldini L and Comoglio PM: Targeting the tumor and its microenvironment by a dual-function decoy Met receptor. Cancer Cell 6: 61-73, 2004.

30. Liu Z, Neiss N, Zhou S, Henne-Bruns D, Korc M, Bachem M and Kornmann M: Identification of a fibroblast growth factor receptor 1 splice variant that inhibits pancreatic cancer cell growth. Cancer Res 67: 2712-2719, 2007.

31. Tiran Z, Oren A, Hermesh C, Rotman G, Levine Z, Amitai H, Handelsman T, Beiman M, Chen A, Landesman-Milo D, Dassa L, Peres Y, Koifman C, Glezer S, Vidal-Finkelstein R, Bahat K, Pergam T, Israel C, Horev J, Tsarfaty I and AyalonSoffer M: A novel recombinant soluble splice variant of Met is a potent antagonist of the hepatocyte growth factor/scatter factorMet pathway. Clin Cancer Res 14: 4612-4621, 2008. 
32. Kong-Beltran M, Stamos J and Wickramasinghe D: The Sema domain of Met is necessary for receptor dimerization and activation. Cancer Cell 6: 75-84, 2004.

33. Boccaccio $\mathrm{C}$ and Comoglio PM: Invasive growth: a MET-driven genetic programme for cancer and stem cells. Nat Rev Cancer 6: 637-645, 2006

34. Xiao ZQ, Chen YQ and Wang MH: Requirement of both tyrosine residues 1330 and 1337 in the C-terminal tail of the RON receptor tyrosine kinase for epithelial cell scattering and migration. Biochem Biophys Res Commun 267: 669-675, 2000

35. Xu XM, Zhou YQ and Wang MH: Mechanisms of cytoplasmic $\{$ beta\}-catenin accumulation and its involvement in tumorigenic activities mediated by oncogenic splicing variant of the receptor originated from Nantes tyrosine kinase. J Biol Chem 280: 25087-25094, 2005
36. Thangasamy A, Rogge J and Ammanamanchi S: Recepteur d'origine nantais tyrosine kinase is a direct target of hypoxiainducible factor-1alpha-mediated invasion of breast carcinoma cells. J Biol Chem 284: 14001-14010, 2009.

37. Wang J, Rajput A, Kan JL, Rose R, Liu XQ, Kuropatwinski K, Hauser J, Beko A, Dominquez I, Sharratt EA, Brattain L, Levea C, Sun FL, Keane DM, Gibson NW and Brattain MG: Knockdown of Ron kinase inhibits mutant phosphatidylinositol 3-kinase and reduces metastasis in human colon carcinoma. J Biol Chem 284: 10912-10922, 2009.

38. Wang MH, Skeel A, Yoshimura T, Copeland TD, Sakaguchi K and Leonard EJ: Antibodies to macrophage stimulating protein (MSP): specificity, epitope interactions, and immunoassay of MSP in human serum. J Leukoc Biol 54: 289-295, 1993. 